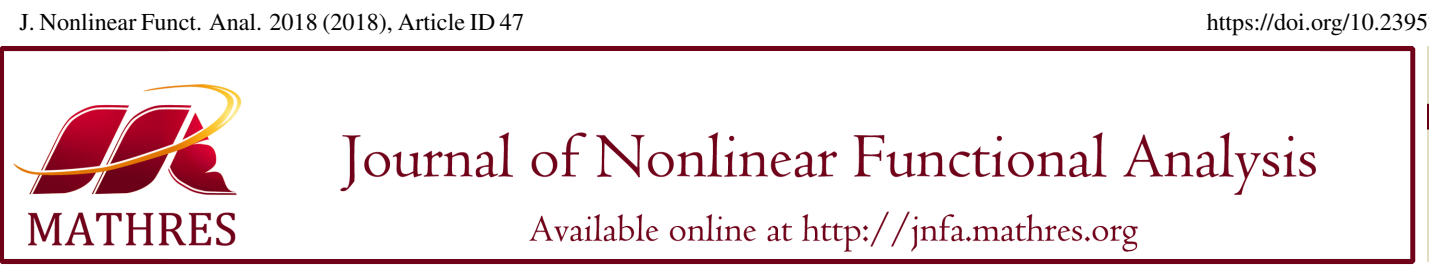

https://doi.org/10.23952/jnfa.2018.47

\title{
SELF-ADAPTIVE ITERATIVE ALGORITHMS FOR SOLVING MULTIPLE-SET SPLIT EQUALITY COMMON FIXED-POINT PROBLEMS OF DEMICONTRACTIVE OPERATORS
}

\author{
HONGYAN SU ${ }^{1}$, JING ZHAO ${ }^{2, *}$ \\ ${ }^{1}$ Department of Mathematics, Qingdao University of Science and Technology, Qingdao 266000, China \\ ${ }^{2}$ College of Science, Civil Aviation University of China, Tianjin 300300, China
}

\begin{abstract}
In this paper, we introduce parallel and cyclic iterative algorithms for solving the multiple-set split equality common fixed-point problem of demicontractive operators. We propose a way of selecting the stepsizes such that the implementation of our algorithms does not need any prior information about operator norms. It thus avoids the difficult task of estimating the operator norms. We also combine the process of cyclic and parallel together and propose two mixed iterative algorithms without prior knowledge of operator norms. The weak convergence theorems of the proposed algorithms are established under some suitable control conditions in a real Hilbert space. Some numerical experiments are given for the proposed iterative algorithms. Keywords. Multiple-set split equality common fixed-point problem; Demicontractive operator; Weak convergence; Iterative algorithm; Hilbert space.
\end{abstract}

2010 Mathematics Subject Classification. 47H09, 47H10, 47J05.

\section{INTRODUCTION}

Throughout this paper, we always assume that $H$ is a real Hilbert space with inner product $\langle\cdot, \cdot\rangle$ and norm $\|\cdot\|$. Let $I$ denote the identity operator on $H$. Let $T: H \rightarrow H$ be an operator. A point $x \in H$ is said to be a fixed point of $T$ provided $T x=x$. In this paper, we use $F(T)$ to denote the fixed-point set of $T$.

Recall that the multiple-sets split feasibility problem (MSFP) which finds application in intensity modulated radiation therapy was proposed in [1] and is formulated as finding a point $x^{*}$ satisfying the property:

$$
x^{*} \in \bigcap_{i=1}^{p} C_{i} \text { such that } A x^{*} \in \bigcap_{j=1}^{r} Q_{j},
$$

where $p, r \geq 1$ are integers, $\left\{C_{i}\right\}_{i=1}^{p}$ and $\left\{Q_{j}\right\}_{j=1}^{r}$ are nonempty closed convex subsets of real Hilbert spaces $H_{1}$ and $H_{2}$, respectively, and $A: H_{1} \rightarrow H_{2}$ is a bounded linear operator. The MSFP (1.1) with

${ }^{*}$ Corresponding author.

E-mail addresses: su_hongyan@163.com (H. Su), zhaojing200103@163.com (J. Zhao).

Received September 20, 2018; Accepted December 14, 2018.

(C)2018 Journal of Nonlinear Functional Analysis 
$p=r=1$ is known as the split feasibility problem (SFP) originally introduced by Censor and Elfving [2], which is defined as follows:

$$
x^{*} \in C \text { such that } A x^{*} \in Q,
$$

where $C$ and $Q$ are nonempty closed convex subset of real Hilbert spaces $H_{1}$ and $H_{2}$, respectively, and $A: H_{1} \rightarrow H_{2}$ is a bounded linear operator. For solving the SFP (1.2), Censor and Elfving [2] used a multidistance method to obtain iterative algorithms, which involved matrix inverses at each step. In order to avoid usage of the inverse, Byrne [3] proposed an iterative method called the CQ algorithm that involves only the orthogonal projections onto $C$ and $Q$ to solve the SFP (1.2). The CQ algorithm is defined as follows:

$$
x_{k+1}=P_{C}\left(I-\gamma A^{*}\left(I-P_{Q}\right) A\right) x_{k}, k \in N,
$$

where $A^{*}$ denotes the adjoint of $A$ and $\gamma \in\left(0, \frac{2}{\lambda}\right)$ with $\lambda$ being the spectral radius of the operator $A^{*} A$. Later, Yang [4] proposed a relaxed CQ algorithm for solving the SFP in which the orthogonal projections $P_{C}$ and $P_{Q}$ are replaced by $P_{C_{n}}$ and $P_{Q_{n}}$, respectively, i.e., the orthogonal projections onto two halfspaces $C_{n}$ and $Q_{n}$. Both the CQ algorithm and the relaxed CQ algorithm used a fixed stepsize related to the largest eigenvalue of $A^{*} A$, which sometimes affects convergence of the algorithms. Qu and Xiu [5] developed the CQ algorithm and the relaxed CQ algorithm by adopting the Armijo-like searches. Some other related results can be found in $[6,7,8,9,10,11,12]$ and references therein.

Since every closed convex subset of a Hilbert space is the fixed point set of its associating projection, the MSFP (1.1) and the SFP (1.2) are all special cases of the so-called multiple-set split common fixedpoint problem (MSCFP), which is formulated as finding a point $x^{*}$ satisfying the property:

$$
x^{*} \in \bigcap_{i=1}^{p} F\left(U_{i}\right) \text { such that } A x^{*} \in \bigcap_{j=1}^{r} F\left(T_{j}\right),
$$

where $p, r \geq 1$ are integers, $\left\{U_{i}\right\}_{i=1}^{p}: H_{1} \rightarrow H_{1}$ and $\left\{T_{j}\right\}_{j=1}^{r}: H_{2} \rightarrow H_{2}$ are nonlinear operators and $A: H_{1} \rightarrow H_{2}$ is a bounded linear operator. In particular, if $p=r=1$, then (1.3) reduces to finding a point $x^{*}$ with the property:

$$
x^{*} \in F(U) \text { such that } A x^{*} \in F(T),
$$

which is usually called the split common fixed-point problem (SCFP). The concept of the SCFP in finite-dimensional Hilbert spaces was originally introduced by Censor and Segal [13]. For nonexpansive operators, they proposed and proved, in finite-dimensional spaces, the convergence of the following algorithm:

$$
x_{k+1}=U\left(x_{k}+\gamma A^{t}(T-I) A x_{k}\right), k \in N,
$$

where $\gamma \in\left(0, \frac{2}{\lambda}\right)$ with $\lambda$ being the largest eigenvalue of $A^{t} A$ ( $A^{t}$ stands for the matrix transposition). Many methods have been proposed for solving the MSCFP and SCFP or their particular cases; see, for example, $[14,15,16,17,18,19,20,21,22]$ and the references therein.

For solving the multiple-set common fixed-point problem, some authors proposed the parallel and cyclic iterative algorithms. Censor and Segal [13] introduced the following parallel iterative algorithm to solve MSCFP (1.3) of directed operators:

$$
x_{k+1}=x_{k}-\gamma\left[\sum_{i=1}^{p} \alpha_{i}\left(x_{k}-U_{i}\left(x_{k}\right)\right)+\sum_{j=1}^{r} \beta_{j} A^{*}\left(A x_{k}-T_{j}\left(A x_{k}\right)\right)\right],
$$


where $\left\{\alpha_{i}\right\}_{i=1}^{p},\left\{\beta_{j}\right\}_{j=1}^{r}$ are nonnegative constants, $0<\gamma<2 / L$ with $L=\sum_{i=1}^{p} \alpha_{i}+\lambda \sum_{j=1}^{r} \beta_{j}$ and $\lambda$ being the largest eigenvalue of $A^{*} A$. Wang and $\mathrm{Xu}$ [23] proved the convergence of the following cyclic iterative algorithm for MSCFP (1.3) of directed operators:

$$
x_{k+1}=U_{[k]_{1}}\left(x_{k}+\gamma A^{*}\left(T_{[k]_{2}}-I\right) A x_{k}\right),
$$

where $0<\gamma<2 / \rho\left(A^{*} A\right),[k]_{1}:=k(\bmod p)$ and $[k]_{2}:=k(\bmod r)$.

For solving MSCFP (1.3) of directed operators, Tang and Liu [24] introduced inner parallel and outer cyclic iterative algorithm:

$$
x_{k+1}=U_{[k]_{1}}\left(x_{k}+\gamma_{k} \Sigma_{j=1}^{r} \eta_{j} A^{*}\left(T_{j}-I\right) A x_{k}\right)
$$

and outer parallel and inner cyclic iterative algorithm:

$$
x_{k+1}=\Sigma_{j=1}^{r} \omega_{i} U_{i}\left(x_{k}+\gamma_{k} A^{*}\left(T_{[k]_{2}}-I\right) A x_{k}\right),
$$

where $[k]_{1}:=k(\bmod p)$ and $[k]_{2}:=k(\bmod r)$.

In [25], Moudafi proposed an algorithm to solve the SCFP when operators $U$ and $T$ are demicontractive. The class of demicontractive operators is fundamental because many common types of operators arising in optimization belong to this class (see Remark 2.3 below). Moudafi proved that the sequence $\left\{x_{k}\right\}$ generated by the following algorithm converges weakly to the solution of SCFP (1.4):

$$
x_{k+1}=\left(1-\alpha_{k}\right) u_{k}+\alpha_{k} U\left(u_{k}\right),
$$

where $u_{k}=x_{k}+\gamma A^{*}(T-I) A x_{k}, \gamma \in\left(0, \frac{1-\mu}{\lambda}\right)$ with $\lambda$ being the spectral radius of the operator $A^{*} A$ and $\left\{\alpha_{k}\right\} \subseteq(0,1)$. For solving MSCFP (1.3) of the demicontractive mappings, Tang, Peng and Liu [26] proposed the following cyclic algorithm and proved the weak convergence under the suitable conditions:

$$
x_{k+1}=\left(1-\alpha_{k}\right) u_{k}+\alpha_{k} U_{i(k)}\left(u_{k}\right),
$$

where $u_{k}=x_{k}+\gamma A^{*}\left(T_{j(k)}-I\right) A x_{k}, i(k)=k(\bmod p)+1, j(k)=k(\bmod r)+1, \gamma \in\left(0, \frac{1-\mu}{\lambda}\right)$ with $\lambda$ being the spectral radius of the operator $A^{*} A$ and $\left\{\alpha_{k}\right\} \subseteq(0,1)$.

Recently, Moudafi [27] introduced the split equality common fixed-point problem (SECFP). Let $H_{1}$, $H_{2}, H_{3}$ be real Hilbert spaces, let $A: H_{1} \rightarrow H_{3}, B: H_{2} \rightarrow H_{3}$ be two bounded linear operators, let $U$ : $H_{1} \rightarrow H_{1}$ and $T: H_{2} \rightarrow H_{2}$ be two firmly quasi-nonexpansive operators. The SECFP in [27] is to

$$
\text { find } x^{*} \in F(U), y^{*} \in F(T) \text { such that } A x^{*}=B y^{*} \text {. }
$$

If $H_{2}=H_{3}$ and $B=I$, then SECFP (1.5) reduces to SCFP (1.4).

For solving SECFP (1.5) of firmly quasi-nonexpansive operators, Moudafi [27] introduced the following alternating algorithm:

$$
\left\{\begin{array}{l}
x_{k+1}=U\left(x_{k}-\gamma_{k} A^{*}\left(A x_{k}-B y_{k}\right)\right), \\
y_{k+1}=T\left(y_{k}+\gamma_{k} B^{*}\left(A x_{k+1}-B y_{k}\right)\right),
\end{array}\right.
$$

where non-decreasing sequence $\gamma_{k} \in\left(\varepsilon, \min \left(\frac{1}{\lambda_{A}}, \frac{1}{\lambda_{B}}\right)-\varepsilon\right), \lambda_{A}, \lambda_{B}$ stand for the spectral radiuses of $A^{*} A$ and $B^{*} B$, respectively.

In [28], Moudafi and Al-Shemas introduced the following simultaneous iterative method to solve SECFP (1.5) of firmly quasi-nonexpansive operators:

$$
\left\{\begin{array}{l}
x_{k+1}=U\left(x_{k}-\gamma_{k} A^{*}\left(A x_{k}-B y_{k}\right)\right), \\
y_{k+1}=T\left(y_{k}+\gamma_{k} B^{*}\left(A x_{k}-B y_{k}\right)\right),
\end{array}\right.
$$


where $\gamma_{k} \in\left(\varepsilon, \frac{2}{\lambda_{A}+\lambda_{B}}-\varepsilon\right), \lambda_{A}$ and $\lambda_{B}$ stand for the spectral radiuses of $A^{*} A$ and $B^{*} B$, respectively.

Let $H_{1}, H_{2}, H_{3}$ be real Hilbert spaces. let $A: H_{1} \rightarrow H_{3}, B: H_{2} \rightarrow H_{3}$ be two bounded linear operators. Let $U_{i}: H_{1} \rightarrow H_{1}(1 \leq i \leq p)$ and $T_{j}: H_{2} \rightarrow H_{2}(1 \leq j \leq r)$ be nonlinear operators. In this paper, inspired and motivated by the works mentioned above, we consider the following multiple-set split equality common fixed-point problem (MSECFP):

$$
\text { finding } x^{*} \in \bigcap_{i=1}^{p} F\left(U_{i}\right), y^{*} \in \bigcap_{j=1}^{r} F\left(T_{j}\right) \text {, such that } A x^{*}=B y^{*} .
$$

This allows asymmetric and partial relations between $x$ and $y$. The interest is to cover many situations, for instance, in decomposition methods for PDEs, applications in game theory and in intensity-modulated radiation therapy (IMRT). In decision sciences, this allows to consider agents who interact only via some components of their decision variables (see [29]). In IMRT, this amounts to envisaging a weak coupling between the vector of doses absorbed in all voxels and that of the radiation intensity (see [30]). Some iterative algorithms have been proposed to solve the SECFP and MSECFP; see, for example, $[31,32,33,34,35]$ and the references therein. In this paper, we propose the parallel and cyclic iterative algorithms to solve MSECFP (1.8) of demicontractive operators. We also propose two mixed iterative algorithms which combine the process of cyclic and parallel together. We introduce a way of selecting the stepsizes such that the implementation of our algorithms does not need any prior information about the operator norms of $A$ and $B$, and the convergence is still guaranteed.

The organization of this paper is as follows. Some useful definitions and results are listed for the convergence analysis of the self-adaptive iterative algorithms in Section 2. In Section 3, we introduce self-adaptive parallel and cyclic iterative algorithms and the weak convergence theorems of the proposed iterative algorithms is obtained. In Section 4, we propose two mixed self-adaptive iterative algorithms which combine the process of cyclic and parallel together and obtain the weak convergence results. Some numerical experiments are provided to illustrate the efficiency of the proposed iterative algorithms in Section 5, the last section.

\section{PRELIMINARIES}

In this paper, let $H_{1}, H_{2}$ and $H_{3}$ be real Hilbert spaces and $\Gamma$ the solution set of MSECFP (1.8). Assume that $A \neq 0$ or $B \neq 0$. We denote by $\omega_{w}\left(x_{k}\right)=\left\{x: \exists x_{k_{j}} \rightarrow x\right\}$ the weak $\omega$-limit set of $\left\{x_{k}\right\}$. We denote the strong convergence and weak convergence by $\rightarrow$ and $\rightarrow$, respectively.

Definition 2.1. An operator $T: H \rightarrow H$ is said to be

(i) nonexpansive if $\|T x-T y\| \leq\|x-y\|$ for all $x, y \in H$;

(ii) quasi-nonexpansive if $F(T) \neq \emptyset$ and $\|T x-q\| \leq\|x-q\|$ for all $x \in H$ and $q \in F(T)$;

(iii) firmly nonexpansive if $\|T x-T y\|^{2} \leq\|x-y\|^{2}-\|(x-y)-(T x-T y)\|^{2}$ for all $x, y \in H$;

(iv) firmly quasi-nonexpansive (also called directed operator) if $F(T) \neq \emptyset$ and $\|T x-q\|^{2} \leq\|x-q\|^{2}-$ $\|x-T x\|^{2}$ for all $x \in H$ and $q \in F(T)$;

(v) $k$-strictly pseudocontractive if there exists a constant $k \in(0,1)$ such that

$$
\|T x-T y\|^{2} \leq\|x-y\|^{2}+k\|(I-T) x-(I-T) y\|^{2}
$$

for all $x, y \in H$; 
(vi) $\beta$-demicontractive if $F(T) \neq \emptyset$ and there exists a constant $\beta \in(0,1)$ such that

$$
\|T x-q\|^{2} \leq\|x-q\|^{2}+\beta\|x-T x\|^{2}
$$

for all $x \in H$ and $q \in F(T)$.

Two equivalent definitions of demicontractive operator are given by the following lemma.

Lemma 2.2. [26] Let $T: H \rightarrow H$ be $\beta$-demicontractive operator. Then the following inequalities are equivalent:

(i) $\langle x-T x, x-q\rangle \geq \frac{1-\beta}{2}\|x-T x\|^{2}, q \in F(T), x \in H$;

(ii) $\langle x-T x, q-T x\rangle \leq \frac{1+\beta}{2}\|x-T x\|^{2}, q \in F(T), x \in H$.

Remark 2.3. It is easy to see that the class of demicontractive operators contain the class of firmly quasi-nonexpansive operators, the class of quasi-nonexpansive operators and the class of strictly pseudocontractive operators with a nonempty fixed point.

Definition 2.4. An operator $T: H \rightarrow H$ is said to be demiclosed at the origin if, for any sequence $\left\{x_{n}\right\}$ with $x_{n} \rightarrow x$, and $T x_{n} \rightarrow 0$, then $T x=0$.

In real Hilbert spaces, we easily get the following equality:

$$
2\langle x, y\rangle=\|x\|^{2}+\|y\|^{2}-\|x-y\|^{2}=\|x+y\|^{2}-\|x\|^{2}-\|y\|^{2}, \forall x, y \in H .
$$

Lemma 2.5. [36] Let $H$ be a real Hilbert space. For each $x_{1}, \ldots, x_{m} \in H$ and $\alpha_{1}, \ldots, \alpha_{m} \in[0,1]$ with $\sum_{i=1}^{m} \alpha_{i}=1$, we have

$$
\left\|\alpha_{1} x_{1}+\alpha_{2} x_{2}+\cdots+\alpha_{m} x_{m}\right\|^{2}=\Sigma_{i=1}^{m} \alpha_{i}\left\|x_{i}\right\|^{2}-\sum_{1 \leq i<j \leq m} \alpha_{i} \alpha_{j}\left\|x_{i}-x_{j}\right\|^{2} .
$$

Now, we give the following property of the relaxed operator $T_{\alpha}=(1-\alpha) I+\alpha T$ for demicontractive operators, which will be needed in our main results.

Lemma 2.6. [25] Let $T$ be a $\beta$-demicontractive operator with $F(T) \neq \emptyset$. Set $T_{\alpha}=(1-\alpha) I+\alpha T$, for $\alpha \in[0,1-\beta]$. Then, $T_{\alpha}$ is quasi-nonexpansive and

$$
\left\|T_{\alpha} x-q\right\|^{2} \leq\|x-q\|^{2}-\alpha(1-\beta-\alpha)\|T x-x\|^{2}, x \in H, q \in F(T) .
$$

Remark 2.7. It is easy to check that $F(T)=F\left(T_{\alpha}\right)$. Hence, $F(T)$ is a closed convex subset of $H$, as the fixed-point set of a quasi-nonexpansive operator.

Lemma 2.8. [37] Let $C$ be a nonempty closed convex subset of a real Hilbert space $H$, and let $T: C \rightarrow C$ be a $k$-strictly pseudocontractive operator. Then I-T is demiclosed at 0.

\section{SELF-ADAPTIVE PARALLEL AND CYCLIC ITERATIVE ALGORITHMS FOR THE MSECFP}

In this section, we introduce two self-adaptive parallel and cyclic iterative algorithms for solving MSECFP (1.8) of demicontractive operators, where stepsizes don't depend on the operator norms $\|A\|$ and $\|B\|$. We prove the weak convergence of the proposed algorithms. Let $\left\{U_{i}\right\}_{i=1}^{p}$ and $\left\{T_{j}\right\}_{j=1}^{r}$ be a finite family of demicontractive operators, i.e., there exists $\left\{\beta_{i}\right\}_{i=1}^{p} \subset(0,1)$ and $\left\{\mu_{j}\right\}_{j=1}^{r} \subset(0,1)$ such that

$$
\left\|U_{i} x-v\right\|^{2} \leq\|x-v\|^{2}+\beta_{i}\left\|x-U_{i} x\right\|^{2}, x \in H_{1}, \quad v \in F\left(U_{i}\right), \quad 1 \leq i \leq p,
$$


and

$$
\left\|T_{j} y-q\right\|^{2} \leq\|y-q\|^{2}+\mu_{j}\left\|y-T_{j} y\right\|^{2}, \quad y \in H_{2}, q \in F\left(T_{j}\right), \quad 1 \leq j \leq r .
$$

Let $\beta=\max _{1 \leq i \leq p}\left\{\beta_{i}\right\}$ and $\mu=\max _{1 \leq j \leq r}\left\{\mu_{j}\right\}$. Then

$$
\left\|U_{i} x-v\right\|^{2} \leq\|x-v\|^{2}+\beta\left\|x-U_{i} x\right\|^{2}, \quad x \in H_{1}, \quad v \in F\left(U_{i}\right), \quad 1 \leq i \leq p,
$$

and

$$
\left\|T_{j} y-q\right\|^{2} \leq\|y-q\|^{2}+\mu\left\|y-T_{j} y\right\|^{2}, \quad y \in H_{2}, \quad q \in F\left(T_{j}\right), \quad 1 \leq j \leq r .
$$

Algorithm 3.1. Let $\left\{\alpha_{k}^{i}\right\}_{k=0}^{\infty} \subset[0,1](0 \leq i \leq p)$ and $\left\{\sigma_{k}^{j}\right\}_{k=0}^{\infty} \subset[0,1](0 \leq j \leq r)$ be sequences such that $\Sigma_{i=0}^{p} \alpha_{k}^{i}=1$ and $\sum_{j=0}^{r} \sigma_{k}^{j}=1$ for every $k \geq 0$. Take $x_{0} \in H_{1}, y_{0} \in H_{2}$ and calculate

$$
\left\{\begin{array}{l}
s_{k}=x_{k}-\gamma_{k} A^{*}\left(A x_{k}-B y_{k}\right) \\
x_{k+1}=\alpha_{k}^{0} s_{k}+\alpha_{k}^{1} U_{1}\left(s_{k}\right)+\cdots+\alpha_{k}^{p} U_{p}\left(s_{k}\right), \\
t_{k}=y_{k}+\gamma_{k} B^{*}\left(A x_{k}-B y_{k}\right) \\
y_{k+1}=\sigma_{k}^{0} t_{k}+\sigma_{k}^{1} T_{1}\left(t_{k}\right)+\cdots+\sigma_{k}^{r} T_{r}\left(t_{k}\right) .
\end{array}\right.
$$

Assume the stepsize $\gamma_{k}$ is chosen in such a way that

$$
\gamma_{k}:= \begin{cases}\frac{\rho_{k}\left\|A x_{k}-B y_{k}\right\|^{2}}{\left\|A^{*}\left(A x_{k}-B y_{k}\right)\right\|^{2}+\left\|B^{*}\left(A x_{k}-B y_{k}\right)\right\|^{2}}, & A x_{k}-B y_{k} \neq 0, \\ \gamma, & A x_{k}-B y_{k}=0\end{cases}
$$

with $0<\liminf _{k \rightarrow \infty} \rho_{k} \leq \limsup \sup _{k \rightarrow \infty} \rho_{k}<2$ and $\gamma>0$.

Lemma 3.2. Assume that $\Gamma$ is nonempty. Then $\gamma_{k}$ defined by (3.2) is well-defined.

Proof. Taking $(x, y) \in \Gamma$, i.e., $x \in \cap_{i=1}^{p} F\left(U_{i}\right), y \in \cap_{j=1}^{r} F\left(T_{j}\right)$ and $A x=B y$, we have

$$
\left\langle A^{*}\left(A x_{k}-B y_{k}\right), x_{k}-x\right\rangle=\left\langle A x_{k}-B y_{k}, A x_{k}-A x\right\rangle
$$

and

$$
\left\langle B^{*}\left(A x_{k}-B y_{k}\right), y-y_{k}\right\rangle=\left\langle A x_{k}-B y_{k}, B y-B y_{k}\right\rangle .
$$

From $A x=B y$, adding the two above equalities, we obtain

$$
\begin{aligned}
\left\|A x_{k}-B y_{k}\right\|^{2} & =\left\langle A^{*}\left(A x_{k}-B y_{k}\right), x_{k}-x\right\rangle+\left\langle B^{*}\left(A x_{k}-B y_{k}\right), y-y_{k}\right\rangle \\
& \leq\left\|A^{*}\left(A x_{k}-B y_{k}\right)\right\| \cdot\left\|x_{k}-x\right\|+\left\|B^{*}\left(A x_{k}-B y_{k}\right)\right\| \cdot\left\|y-y_{k}\right\| .
\end{aligned}
$$

For $\left\|A x_{k}-B y_{k}\right\|>0$, we have $\left\|A^{*}\left(A x_{k}-B y_{k}\right)\right\| \neq 0$ or $\left\|B^{*}\left(A x_{k}-B y_{k}\right)\right\| \neq 0$. So $\gamma_{k}$ is well-defined. This completes the proof.

Theorem 3.3. Let $\left\{U_{i}, \quad 1 \leq i \leq p\right\}: H_{1} \rightarrow H_{1}$ and $\left\{T_{j}, \quad 1 \leq j \leq r\right\}: H_{2} \rightarrow H_{2}$ be $\beta_{i}$-demicontractive and $\mu_{j}$-demicontractive operators, respectively. Assume that $U_{i}-I(1 \leq i \leq p), T_{j}-I(1 \leq j \leq r)$ are demiclosed at origin and $\Gamma$ is nonempty. Then the sequence $\left\{\left(x_{k}, y_{k}\right)\right\}$ generated by Algorithm 3.1 weakly converges to a solution $\left(x^{*}, y^{*}\right)$ of (1.8), provided that $\liminf _{k \rightarrow \infty} \alpha_{k}^{0}>\beta, \liminf _{k \rightarrow \infty} \alpha_{k}^{i}>0, \forall 1 \leq i \leq p$ and $\liminf _{k \rightarrow \infty} \sigma_{k}^{0}>\mu, \liminf _{k \rightarrow \infty} \sigma_{k}^{j}>0, \forall 1 \leq j \leq r$. Moreover, $\left\|A x_{k}-B y_{k}\right\| \rightarrow 0,\left\|x_{k}-x_{k+1}\right\| \rightarrow 0$ and $\left\|y_{k}-y_{k+1}\right\| \rightarrow 0$ as $k \rightarrow \infty$. 
Proof. First, for any $\left(x^{*}, y^{*}\right) \in \Gamma$, we show that $\lim _{k \rightarrow \infty}\left(\left\|x_{k}-x^{*}\right\|^{2}+\left\|y_{k}-y^{*}\right\|^{2}\right)$ exists. Taking $\left(x^{*}, y^{*}\right) \in$ $\Gamma$, i.e., $x^{*} \in \bigcap_{i=1}^{p} F\left(U_{i}\right), y^{*} \in \bigcap_{j=1}^{r} F\left(T_{j}\right)$ and $A x^{*}=B y^{*}$, we have

$$
\begin{aligned}
& \left\|s_{k}-x^{*}\right\|^{2} \\
= & \left\|x_{k}-\gamma_{k} A^{*}\left(A x_{k}-B y_{k}\right)-x^{*}\right\|^{2} \\
= & \left\|x_{k}-x^{*}\right\|^{2}-2 \gamma_{k}\left\langle x_{k}-x^{*}, A^{*}\left(A x_{k}-B y_{k}\right)\right\rangle+\gamma_{k}^{2}\left\|A^{*}\left(A x_{k}-B y_{k}\right)\right\|^{2} .
\end{aligned}
$$

By (2.1), we find that

$$
\begin{aligned}
& -2\left\langle x_{k}-x^{*}, A^{*}\left(A x_{k}-B y_{k}\right)\right\rangle \\
= & -2\left\langle A x_{k}-A x^{*}, A x_{k}-B y_{k}\right\rangle \\
= & -\left\|A x_{k}-A x^{*}\right\|^{2}-\left\|A x_{k}-B y_{k}\right\|^{2}+\left\|B y_{k}-A x^{*}\right\|^{2} .
\end{aligned}
$$

From (3.4) and (3.5), we obtain that

$$
\begin{aligned}
\left\|s_{k}-x^{*}\right\|^{2}= & \left\|x_{k}-x^{*}\right\|^{2}-\gamma_{k}\left\|A x_{k}-B y_{k}\right\|^{2}-\gamma_{k}\left\|A x_{k}-A x^{*}\right\|^{2} \\
& +\gamma_{k}\left\|B y_{k}-A x^{*}\right\|^{2}+\gamma_{k}^{2}\left\|A^{*}\left(A x_{k}-B y_{k}\right)\right\|^{2} .
\end{aligned}
$$

Similarly, we have

$$
\begin{aligned}
\left\|t_{k}-y^{*}\right\|^{2}= & \left\|y_{k}-y^{*}\right\|^{2}-\gamma_{k}\left\|A x_{k}-B y_{k}\right\|^{2}-\gamma_{k}\left\|B y_{k}-B y^{*}\right\|^{2} \\
& +\gamma_{k}\left\|A x_{k}-B y^{*}\right\|^{2}+\gamma_{k}^{2}\left\|B^{*}\left(A x_{k}-B y_{k}\right)\right\|^{2} .
\end{aligned}
$$

Noting $A x^{*}=B y^{*}$, we see that

$$
\begin{aligned}
& \left\|s_{k}-x^{*}\right\|^{2}+\left\|t_{k}-y^{*}\right\|^{2} \\
\leq & \left\|x_{k}-x^{*}\right\|^{2}+\left\|y_{k}-y^{*}\right\|^{2}-\gamma_{k}\left[2\left\|A x_{k}-B y_{k}\right\|^{2}-\gamma_{k}\left(\left\|A^{*}\left(A x_{k}-B y_{k}\right)\right\|^{2}+\left\|B^{*}\left(A x_{k}-B y_{k}\right)\right\|^{2}\right)\right] .
\end{aligned}
$$

By using (3.2), we find that

$$
\left\|s_{k}-x^{*}\right\|^{2}+\left\|t_{k}-y^{*}\right\|^{2} \leq\left\|x_{k}-x^{*}\right\|^{2}+\left\|y_{k}-y^{*}\right\|^{2} .
$$

Since $U_{i}$ is $\beta_{i}$-demicontractive $(1 \leq i \leq p)$, from Lemma 2.5, we have

$$
\begin{aligned}
& \left\|x_{k+1}-x^{*}\right\|^{2} \\
\leq & \alpha_{k}^{0}\left\|s_{k}-x^{*}\right\|^{2}+\sum_{i=1}^{p} \alpha_{k}^{i}\left\|U_{i}\left(s_{k}\right)-x^{*}\right\|^{2}-\sum_{i=1}^{p} \alpha_{k}^{0} \alpha_{k}^{i}\left\|U_{i}\left(s_{k}\right)-s_{k}\right\|^{2} \\
\leq & \alpha_{k}^{0}\left\|s_{k}-x^{*}\right\|^{2}+\sum_{i=1}^{p} \alpha_{k}^{i}\left(\left\|s_{k}-x^{*}\right\|^{2}+\beta\left\|U_{i}\left(s_{k}\right)-s_{k}\right\|^{2}\right)-\sum_{i=1}^{p} \alpha_{k}^{0} \alpha_{k}^{i}\left\|U_{i}\left(s_{k}\right)-s_{k}\right\|^{2} \\
= & \left\|s_{k}-x^{*}\right\|^{2}-\left(\alpha_{k}^{0}-\beta\right)\left(\sum_{i=1}^{p} \alpha_{k}^{i}\left\|U_{i}\left(s_{k}\right)-s_{k}\right\|^{2}\right) .
\end{aligned}
$$

Similarly, since $T_{j}$ is $\mu_{j}$-demicontractive $(1 \leq j \leq r)$, we can get

$$
\left\|y_{k+1}-y^{*}\right\|^{2} \leq\left\|t_{k}-y^{*}\right\|^{2}-\left(\sigma_{k}^{0}-\mu\right)\left(\sum_{j=1}^{r} \sigma_{k}^{j}\left\|T_{j}\left(t_{k}\right)-t_{k}\right\|^{2}\right) .
$$


Adding up (3.10) and (3.11), and setting $l_{k}\left(x^{*}, y^{*}\right)=\left\|x_{k}-x^{*}\right\|^{2}+\left\|y_{k}-y^{*}\right\|^{2}$, we get

$$
\begin{aligned}
& l_{k+1}\left(x^{*}, y^{*}\right) \\
& \leq l_{k}\left(x^{*}, y^{*}\right)-\left(\alpha_{k}^{0}-\beta\right)\left(\sum_{i=1}^{p} \alpha_{k}^{i}\left\|U_{i}\left(s_{k}\right)-s_{k}\right\|^{2}\right)-\left(\sigma_{k}^{0}-\mu\right)\left(\sum_{j=1}^{r} \sigma_{k}^{j}\left\|T_{j}\left(t_{k}\right)-t_{k}\right\|^{2}\right) \\
& -\gamma_{k}\left[2\left\|A x_{k}-B y_{k}\right\|^{2}-\gamma_{k}\left(\left\|A^{*}\left(A x_{k}-B y_{k}\right)\right\|^{2}+\left\|B^{*}\left(A x_{k}-B y_{k}\right)\right\|^{2}\right)\right] .
\end{aligned}
$$

For the case $A x_{k}-B y_{k}=0$, we have

$$
l_{k+1}\left(x^{*}, y^{*}\right) \leq l_{k}\left(x^{*}, y^{*}\right)-\left(\alpha_{k}^{0}-\beta\right)\left(\sum_{i=1}^{p} \alpha_{k}^{i}\left\|U_{i}\left(s_{k}\right)-s_{k}\right\|^{2}\right)-\left(\sigma_{k}^{0}-\mu\right)\left(\sum_{j=1}^{r} \sigma_{k}^{j}\left\|T_{j}\left(t_{k}\right)-t_{k}\right\|^{2}\right) .
$$

Otherwise, we deduce from (3.2) and (3.12) that

$$
\begin{aligned}
l_{k+1}\left(x^{*}, y^{*}\right) & \leq l_{k}\left(x^{*}, y^{*}\right)-\left(\alpha_{k}^{0}-\beta\right)\left(\sum_{i=1}^{p} \alpha_{k}^{i}\left\|U_{i}\left(s_{k}\right)-s_{k}\right\|^{2}\right) \\
& -\left(\sigma_{k}^{0}-\mu\right)\left(\sum_{j=1}^{r} \sigma_{k}^{j}\left\|T_{j}\left(t_{k}\right)-t_{k}\right\|^{2}\right) \\
& -\rho_{k}\left(2-\rho_{k}\right) \frac{\left\|A x_{k}-B y_{k}\right\|^{4}}{\left\|A^{*}\left(A x_{k}-B y_{k}\right)\right\|^{2}+\left\|B^{*}\left(A x_{k}-B y_{k}\right)\right\|^{2}} .
\end{aligned}
$$

By (3.13) and (3.14), we see that sequence $\left\{l_{k}\left(x^{*}, y^{*}\right)\right\}$ is non-increasing and lower bounded by 0. So, $\left\{l_{k}\left(x^{*}, y^{*}\right)\right\}$ converges to some finite limit, say, $l\left(x^{*}, y^{*}\right)$. Hence, $\left\{x_{k}\right\}$ and $\left\{y_{k}\right\}$ are bounded.

Next, we show that

$$
\lim _{k \rightarrow \infty}\left\|A x_{k}-B y_{k}\right\|=\lim _{k \rightarrow \infty}\left\|s_{k}-U_{i}\left(s_{k}\right)\right\|=\lim _{k \rightarrow \infty}\left\|t_{k}-T_{j}\left(t_{k}\right)\right\|=0
$$

for each $1 \leq i \leq p$ and $1 \leq j \leq r$. From (3.14), for $A x_{k}-B y_{k} \neq 0$, we have

$$
\rho_{k}\left(2-\rho_{k}\right) \frac{\left\|A x_{k}-B y_{k}\right\|^{4}}{\left\|A^{*}\left(A x_{k}-B y_{k}\right)\right\|^{2}+\left\|B^{*}\left(A x_{k}-B y_{k}\right)\right\|^{2}} \leq l_{k}\left(x^{*}, y^{*}\right)-l_{k+1}\left(x^{*}, y^{*}\right),
$$

which implies that

$$
\lim _{k \rightarrow \infty} \frac{\left\|A x_{k}-B y_{k}\right\|^{4}}{\left\|A^{*}\left(A x_{k}-B y_{k}\right)\right\|^{2}+\left\|B^{*}\left(A x_{k}-B y_{k}\right)\right\|^{2}}=0
$$

with the assumption on $\left\{\rho_{k}\right\}$. Similarly, by the conditions on $\left\{\alpha_{k}^{i}\right\}(0 \leq i \leq p)$ and $\left\{\sigma_{k}^{j}\right\}(0 \leq j \leq r)$, we obtain that, for each $1 \leq i \leq p, 1 \leq j \leq r$,

$$
\lim _{k \rightarrow \infty}\left\|s_{k}-U_{i}\left(s_{k}\right)\right\|=0
$$

and

$$
\lim _{k \rightarrow \infty}\left\|t_{k}-T_{j}\left(t_{k}\right)\right\|=0
$$

If $A x_{k}-B y_{k}=0$, it is clear that

$$
s_{k}-x_{k}=\gamma_{k} A^{*}\left(A x_{k}-B y_{k}\right)=0 .
$$


Otherwise, it follows from (3.15) that

$$
\begin{aligned}
\frac{1}{\|A\|^{2}+\|B\|^{2}}\left\|A x_{k}-B y_{k}\right\|^{2} & =\left\|A x_{k}-B y_{k}\right\|^{2} \frac{\left\|A x_{k}-B y_{k}\right\|^{2}}{\left(\|A\|^{2}+\|B\|^{2}\right)\left\|A x_{k}-B y_{k}\right\|^{2}} \\
& \leq\left\|A x_{k}-B y_{k}\right\|^{2} \frac{\left\|A x_{k}-B y_{k}\right\|^{2}}{\left\|A^{*}\left(A x_{k}-B y_{k}\right)\right\|^{2}+\left\|B^{*}\left(A x_{k}-B y_{k}\right)\right\|^{2}} \\
& =\frac{\left\|A x_{k}-B y_{k}\right\|^{4}}{\left\|A^{*}\left(A x_{k}-B y_{k}\right)\right\|^{2}+\left\|B^{*}\left(A x_{k}-B y_{k}\right)\right\|^{2}} \rightarrow 0,
\end{aligned}
$$

which implies that

$$
\lim _{k \rightarrow \infty}\left\|A x_{k}-B y_{k}\right\|=0
$$

Moreover, from (3.15), we also have

$$
\begin{aligned}
& \frac{\left\|A x_{k}-B y_{k}\right\|^{2}}{\max \left\{\left\|A^{*}\left(A x_{k}-B y_{k}\right)\right\|,\left\|B^{*}\left(A x_{k}-B y_{k}\right)\right\|\right\}} \\
& =\sqrt{\frac{\left\|A x_{k}-B y_{k}\right\|^{4}}{\max \left\{\left\|A^{*}\left(A x_{k}-B y_{k}\right)\right\|^{2},\left\|B^{*}\left(A x_{k}-B y_{k}\right)\right\|^{2}\right\}}} \\
& \leq \sqrt{\frac{2\left\|A x_{k}-B y_{k}\right\|^{4}}{\left\|A^{*}\left(A x_{k}-B y_{k}\right)\right\|^{2}+\left\|B^{*}\left(A x_{k}-B y_{k}\right)\right\|^{2}}} \rightarrow 0 .
\end{aligned}
$$

So, we have

$$
\begin{aligned}
\left\|s_{k}-x_{k}\right\| & =\left\|\gamma_{k} A^{*}\left(A x_{k}-B y_{k}\right)\right\| \\
& =\frac{\rho_{k}\left\|A x_{k}-B y_{k}\right\|^{2}}{\left\|A^{*}\left(A x_{k}-B y_{k}\right)\right\|^{2}+\left\|B^{*}\left(A x_{k}-B y_{k}\right)\right\|^{2}}\left\|A^{*}\left(A x_{k}-B y_{k}\right)\right\| \\
& \leq \frac{\rho_{k}\left\|A x_{k}-B y_{k}\right\|^{2} \max \left\{\left\|A^{*}\left(A x_{k}-B y_{k}\right)\right\|,\left\|B^{*}\left(A x_{k}-B y_{k}\right)\right\|\right\}}{\max \left\{\left\|A^{*}\left(A x_{k}-B y_{k}\right)\right\|^{2},\left\|B^{*}\left(A x_{k}-B y_{k}\right)\right\|^{2}\right\}} \\
& =\frac{\rho_{k}\left\|A x_{k}-B y_{k}\right\|^{2}}{\max \left\{\left\|A^{*}\left(A x_{k}-B y_{k}\right)\right\|,\left\|B^{*}\left(A x_{k}-B y_{k}\right)\right\|\right\}} \rightarrow 0 .
\end{aligned}
$$

It follows from (3.16) that $\lim _{k \rightarrow \infty}\left\|x_{k}-U_{i}\left(s_{k}\right)\right\|=0$ for all $1 \leq i \leq p$. So, from

$$
\left\|x_{k+1}-x_{k}\right\| \leq \alpha_{k}^{0}\left\|s_{k}-x_{k}\right\|+\alpha_{k}^{1}\left\|U_{1}\left(s_{k}\right)-x_{k}\right\|+\cdots+\alpha_{k}^{p}\left\|U_{p}\left(s_{k}\right)-x_{k}\right\|,
$$

we have

$$
\lim _{k \rightarrow \infty}\left\|x_{k+1}-x_{k}\right\|=0
$$

which infers that $\left\{x_{k}\right\}$ is asymptotically regular. Similarly, we have $\lim _{k \rightarrow \infty}\left\|t_{k}-y_{k}\right\|=0$ as $k \rightarrow \infty$ and $\left\{y_{k}\right\}$ is asymptotically regular.

Now, we prove that $\omega_{w}\left(x_{k}, y_{k}\right) \subseteq \Gamma$. Taking $(\tilde{x}, \tilde{y}) \in \omega_{\omega}\left(x_{k}, y_{k}\right)$, from $\lim _{k \rightarrow \infty}\left\|s_{k}-x_{k}\right\|=0$ and $\lim _{k \rightarrow \infty}\left\|t_{k}-y_{k}\right\|=0$, we have $(\tilde{x}, \tilde{y}) \in \omega_{\omega}\left(s_{k}, t_{k}\right)$. For $1 \leq i \leq p$ and $1 \leq j \leq r$, combined with the demiclosednesses of $U_{i}-I$ and $T_{j}-I$ at 0 , we find from (3.16) and (3.17) that $U_{i}(\tilde{x})=\tilde{x}$ and $T_{j}(\tilde{y})=\tilde{y}$. So $\tilde{x} \in \bigcap_{i=1}^{p} F\left(U_{i}\right)$ and $\tilde{y} \in \bigcap_{j=1}^{r} F\left(T_{j}\right)$. On the other hand, $A \tilde{x}-B \tilde{y} \in \omega_{w}\left(A x_{k}-B y_{k}\right)$ and weakly lower semicontinuity of norms imply that

$$
\|A \tilde{x}-B \tilde{y}\| \leq \liminf _{k \rightarrow \infty}\left\|A x_{k}-B y_{k}\right\|=0 .
$$

Hence $(\tilde{x}, \tilde{y}) \in \Gamma$. So $\omega_{w}\left(x_{k}, y_{k}\right) \subseteq \Gamma$. 
Finally, we show the uniquess of the weak cluster point $\left\{\left(x_{k}, y_{k}\right)\right\}$. Indeed, let $(\bar{x}, \bar{y})$ be other weak cluster point of $\left\{\left(x_{k}, y_{k}\right)\right\}$. Then $(\bar{x}, \bar{y}) \in \Gamma$. From the definition of $l_{k}(x, y)$, we have

$$
\begin{aligned}
& l_{k}(\tilde{x}, \tilde{y}) \\
= & \left\|x_{k}-\bar{x}\right\|^{2}+\|\bar{x}-\tilde{x}\|^{2}+2\left\langle x_{k}-\bar{x}, \bar{x}-\tilde{x}\right\rangle+\left\|y_{k}-\bar{y}\right\|^{2}+\|\bar{y}-\tilde{y}\|^{2}+2\left\langle y_{k}-\bar{y}, \bar{y}-\tilde{y}\right\rangle \\
= & l_{k}(\bar{x}, \bar{y})+\|\bar{x}-\tilde{x}\|^{2}+2\left\langle x_{k}-\bar{x}, \bar{x}-\tilde{x}\right\rangle+\|\bar{y}-\tilde{y}\|^{2}+2\left\langle y_{k}-\bar{y}, \bar{y}-\tilde{y}\right\rangle .
\end{aligned}
$$

Without loss of generality, we may assume that $x_{k} \rightarrow \bar{x}$ and $y_{k} \rightarrow \bar{y}$. By passing to the limit in relation (3.22), we obtain that

$$
l(\tilde{x}, \tilde{y})=l(\bar{x}, \bar{y})+\|\bar{x}-\tilde{x}\|^{2}+\|\bar{y}-\tilde{y}\|^{2} .
$$

Reversing the role of $(\tilde{x}, \tilde{y})$ and $(\bar{x}, \bar{y})$, we also have

$$
l(\bar{x}, \bar{y})=l(\tilde{x}, \tilde{y})+\|\tilde{x}-\bar{x}\|^{2}+\|\tilde{y}-\bar{y}\|^{2} .
$$

By adding the two last equalities, we obtain $\tilde{x}=\bar{x}$ and $\tilde{y}=\bar{y}$, which implies that the whole $\left\{\left(x_{k}, y_{k}\right)\right\}$ weakly converges to the solution of (1.8). This completes the proof.

Next, we propose a self-adaptive cyclic iterative algorithm for solving MSECFP (1.8) of demicontractive operators.

Algorithm 3.4. Assume the sequences $\left\{\alpha_{k}\right\} \subset[0,1]$ and $\left\{\sigma_{k}\right\} \subset[0,1]$. Take $x_{0} \in H_{1}, y_{0} \in H_{2}$, and calculate

$$
\left\{\begin{array}{l}
s_{k}=x_{k}-\gamma_{k} A^{*}\left(A x_{k}-B y_{k}\right), \\
x_{k+1}=\left(1-\alpha_{k}\right) s_{k}+\alpha_{k} U_{i(k)}\left(s_{k}\right), \\
t_{k}=y_{k}+\gamma_{k} B^{*}\left(A x_{k}-B y_{k}\right), \\
y_{k+1}=\left(1-\sigma_{k}\right) t_{k}+\sigma_{k} T_{j(k)}\left(t_{k}\right),
\end{array}\right.
$$

where $i(k)=k(\bmod p)+1, j(k)=k(\bmod r)+1$ and the stepsize $\left\{\gamma_{k}\right\}$ is chosen as (3.2).

Theorem 3.5. Let $\left\{U_{i}, 1 \leq i \leq p\right\}: H_{1} \rightarrow H_{1}$ and $\left\{T_{j}, \quad 1 \leq j \leq r\right\}: H_{2} \rightarrow H_{2}$ be $\beta_{i}$-demicontractive and $\mu_{j}$-demicontractive operators, respectively. Assume that $U_{i}-I(1 \leq i \leq p), T_{j}-I(1 \leq j \leq r)$ are demiclosed at origin and $\Gamma$ is nonempty. Then the sequence $\left\{\left(x_{k}, y_{k}\right)\right\}$ generated by Algorithm 3.4 weakly converges to a solution $\left(x^{*}, y^{*}\right)$ of (1.8), provided that $0<\liminf _{k \rightarrow \infty} \alpha_{k} \leq \limsup _{k \rightarrow \infty} \alpha_{k}<1-\beta$ and $0<\liminf _{k \rightarrow \infty} \sigma_{k} \leq \limsup _{k \rightarrow \infty} \sigma_{k}<1-\mu$. Moreover, $\left\|A x_{k}-B y_{k}\right\| \rightarrow 0,\left\|x_{k}-x_{k+1}\right\| \rightarrow 0$ and $\| y_{k}-$ $y_{k+1} \| \rightarrow 0$ as $k \rightarrow \infty$.

Proof. Take $\left(x^{*}, y^{*}\right) \in \Gamma$. Similar to the proof of Theorem 3.3, we obtain that (3.8) and (3.9) hold. Since, for each $1 \leq i \leq p$ and $1 \leq j \leq r, U_{i}$ and $T_{j}$ are demicontractive, from Lemma 2.6, we get

$$
\left\|x_{k+1}-x^{*}\right\|^{2} \leq\left\|s_{k}-x^{*}\right\|^{2}-\alpha_{k}\left(1-\beta-\alpha_{k}\right)\left\|s_{k}-U_{i(k)}\left(s_{k}\right)\right\|^{2}
$$

and

$$
\left\|y_{k+1}-y^{*}\right\|^{2} \leq\left\|t_{k}-y^{*}\right\|^{2}-\sigma_{k}\left(1-\mu-\sigma_{k}\right)\left\|t_{k}-T_{j(k)}\left(t_{k}\right)\right\|^{2} .
$$

Adding up (3.24) and (3.25), and setting $l_{k}\left(x^{*}, y^{*}\right)=\left\|x_{k}-x^{*}\right\|^{2}+\left\|y_{k}-y^{*}\right\|^{2}$, we get

$$
\begin{aligned}
& l_{k+1}\left(x^{*}, y^{*}\right) \\
\leq & l_{k}\left(x^{*}, y^{*}\right)-\alpha_{k}\left(1-\beta-\alpha_{k}\right)\left\|s_{k}-U_{i(k)}\left(s_{k}\right)\right\|^{2}-\sigma_{k}\left(1-\mu-\sigma_{k}\right)\left\|t_{k}-T_{j(k)}\left(t_{k}\right)\right\|^{2} \\
& -\gamma_{k}\left[2\left\|A x_{k}-B y_{k}\right\|^{2}-\gamma_{k}\left(\left\|A^{*}\left(A x_{k}-B y_{k}\right)\right\|^{2}+\left\|B^{*}\left(A x_{k}-B y_{k}\right)\right\|^{2}\right)\right]
\end{aligned}
$$


by (3.8). We see that $\left\{l_{k}\left(x^{*}, y^{*}\right)\right\}$ is non-increasing and lower bounded by 0 . So, $\left\{l_{k}\left(x^{*}, y^{*}\right)\right\}$ converges to some finite limit, say, $l\left(x^{*}, y^{*}\right)$. Similar to the proof of Theorem 3.3, we get

$$
\lim _{k \rightarrow \infty}\left\|A x_{k}-B y_{k}\right\|=\lim _{k \rightarrow \infty}\left\|s_{k}-U_{i(k)}\left(s_{k}\right)\right\|=\lim _{k \rightarrow \infty}\left\|t_{k}-T_{j(k)}\left(t_{k}\right)\right\|=0 .
$$

and

$$
\lim _{k \rightarrow \infty}\left\|s_{k}-x_{k}\right\|=\lim _{k \rightarrow \infty}\left\|t_{k}-y_{k}\right\|=0 .
$$

It follows from (3.27) and (3.28) that

$$
\lim _{k \rightarrow \infty}\left\|x_{k}-U_{i(k)}\left(s_{k}\right)\right\|=\lim _{k \rightarrow \infty}\left\|y_{k}-T_{j(k)}\left(t_{k}\right)\right\|=0 .
$$

From

$$
\left\|x_{k+1}-x_{k}\right\| \leq\left(1-\alpha_{k}\right)\left\|s_{k}-x_{k}\right\|+\alpha_{k}\left\|U_{i(k)}\left(s_{k}\right)-x_{k}\right\|
$$

and

$$
\left\|y_{k+1}-y_{k}\right\| \leq\left(1-\sigma_{k}\right)\left\|t_{k}-y_{k}\right\|+\sigma_{k}\left\|T_{j(k)}\left(t_{k}\right)-y_{k}\right\|,
$$

we have

$$
\lim _{k \rightarrow \infty}\left\|x_{k+1}-x_{k}\right\|=\lim _{k \rightarrow \infty}\left\|y_{k+1}-y_{k}\right\|=0,
$$

which infers that $\left\{x_{k}\right\}$ and $\left\{y_{k}\right\}$ are asymptotically regular. It follows from

$$
\left\|s_{k+1}-s_{k}\right\| \leq\left\|s_{k+1}-x_{k+1}\right\|+\left\|x_{k+1}-x_{k}\right\|+\left\|x_{k}-s_{k}\right\|
$$

and

$$
\left\|t_{k+1}-t_{k}\right\| \leq\left\|t_{k+1}-y_{k+1}\right\|+\left\|y_{k+1}-y_{k}\right\|+\left\|y_{k}-t_{k}\right\|
$$

that

$$
\lim _{k \rightarrow \infty}\left\|s_{k+1}-s_{k}\right\|=\lim _{k \rightarrow \infty}\left\|t_{k+1}-t_{k}\right\|=0 .
$$

So

$$
\lim _{k \rightarrow \infty}\left\|s_{k+i}-s_{k}\right\|=\lim _{k \rightarrow \infty}\left\|t_{k+j}-t_{k}\right\|=0, \quad 1 \leq i \leq p, 1 \leq j \leq r .
$$

Taking $(\tilde{x}, \tilde{y}) \in \omega_{\omega}\left(x_{k}, y_{k}\right)$, we have $(\tilde{x}, \tilde{y}) \in \omega_{\omega}\left(s_{k}, t_{k}\right)$. Let an index $i \in\{1,2, \cdots, p\}$ be fixed. Noting that the pool of indexes is finite, from (3.30), we can find a subsequence $\left\{s_{k_{m}}\right\} \subset\left\{s_{k}\right\}$ such that $s_{k_{m}} \rightarrow \tilde{x}$ as $m \rightarrow \infty$ and $i\left(k_{m}\right)=i$ for all $m \in \mathbb{N}$. It turns out from (3.27) that

$$
\lim _{m \rightarrow \infty}\left\|s_{k_{m}}-U_{i}\left(s_{k_{m}}\right)\right\|=\lim _{m \rightarrow \infty}\left\|s_{k_{m}}-U_{i\left(k_{m}\right)}\left(s_{k_{m}}\right)\right\|=0 .
$$

Combined with the demiclosednesses of $U_{i}-I$ at 0 , it follows from (3.31) that $U_{i}(\tilde{x})=\tilde{x}$. So $\tilde{x} \in F\left(U_{i}\right)$. Hence $\tilde{x} \in \bigcap_{i=1}^{p} F\left(U_{i}\right)$. By the same reason, we get $\tilde{y} \in \bigcap_{j=1}^{r} F\left(T_{j}\right)$. Similar to the proof of Theorem 3.3, we have $\omega_{w}\left(x_{k}, y_{k}\right) \subseteq \Gamma$ and the sequence $\left\{\left(x_{k}, y_{k}\right)\right\}$ weakly converges to the solution of (1.8). This completes the proof.

Remark 3.6. Algorithm 3.1 and Algorithm 3.4 not only extend the iteration methods of Moudafi [28] from the stepsizes relying on operator norms to self-adaptive stepsizes, but also generalize the results of Zhao and He [35] from quasi-nonexpansive operators to demicontractive operators for solving the MSECFP.

Remark 3.7. For the particular case " $\mathrm{p}=\mathrm{r}=1$ ", Algorithm 3.1 and Algorithm 3.4 solve SECFP (1.5) governed by demicontractive operators without prior knowledge of operator norms. 


\section{SELF-ADAPTIVE MIXED ITERATIVE ALGORITHMS FOR THE MSECFP}

In this section, we introduce two mixed parallel and cyclic iterative algorithms for solving MSECFP (1.8) of demicontractive operators where the stepsizes do not depend on the operator norms $\|A\|$ and $\|B\|$ and prove the weak convergence of the algorithms.

Algorithm 4.1. Let $\left\{\alpha_{k}^{i}\right\}_{k=0}^{\infty} \subset[0,1](0 \leq i \leq p)$ be sequences such that $\Sigma_{i=0}^{p} \alpha_{k}^{i}=1$ for every $k \geq 0$ and the sequence $\left\{\sigma_{k}\right\} \subset[0,1]$. Take $x_{0} \in H_{1}, y_{0} \in H_{2}$, and calculate

$$
\left\{\begin{array}{l}
s_{k}=x_{k}-\gamma_{k} A^{*}\left(A x_{k}-B y_{k}\right), \\
x_{k+1}=\alpha_{k}^{0} s_{k}+\alpha_{k}^{1} U_{1}\left(s_{k}\right)+\cdots+\alpha_{k}^{p} U_{p}\left(s_{k}\right), \\
t_{k}=y_{k}+\gamma_{k} B^{*}\left(A x_{k}-B y_{k}\right), \\
y_{k+1}=\left(1-\sigma_{k}\right) t_{k}+\sigma_{k} T_{j(k)}\left(t_{k}\right),
\end{array}\right.
$$

where $j(k)=k(\bmod r)+1$ and the stepsize $\left\{\gamma_{k}\right\}$ is chosen as (3.2).

Theorem 4.2. Let $\left\{U_{i}, \quad 1 \leq i \leq p\right\}: H_{1} \rightarrow H_{1}$ and $\left\{T_{j}, \quad 1 \leq j \leq r\right\}: H_{2} \rightarrow H_{2}$ be $\beta_{i}$-demicontractive and $\mu_{j}$-demicontractive operators, respectively. Assume that $U_{i}-I(1 \leq i \leq p), T_{j}-I(1 \leq j \leq r)$ are demiclosed at origin and $\Gamma$ is nonempty. Let $\beta=\max _{1 \leq i \leq p}\left\{\beta_{i}\right\}$ and $\mu=\max _{1 \leq j \leq r}\left\{\mu_{j}\right\}$. Then the sequence $\left\{\left(x_{k}, y_{k}\right)\right\}$ generated by Algorithm 4.1 weakly converges to a solution $\left(x^{*}, y^{*}\right)$ of $(1.8)$, provided that $\liminf _{k \rightarrow \infty} \alpha_{k}^{0}>\beta, \liminf _{k \rightarrow \infty} \alpha_{k}^{i}>0$ for all $1 \leq i \leq p$ and $0<\liminf _{k \rightarrow \infty} \sigma_{k} \leq \limsup _{k \rightarrow \infty} \sigma_{k}<$ $1-\mu$. Moreover, $\left\|A x_{k}-B y_{k}\right\| \rightarrow 0,\left\|x_{k}-x_{k+1}\right\| \rightarrow 0$ and $\left\|y_{k}-y_{k+1}\right\| \rightarrow 0$ as $k \rightarrow \infty$.

Proof. Taking $\left(x^{*}, y^{*}\right) \in \Gamma$, similar to the proof of Theorem 3.3, we have that (3.8) and (3.9) hold. From the proof of Theorem 3.3, we have (3.10) holds, i.e.,

$$
\left\|x_{k+1}-x^{*}\right\|^{2} \leq\left\|s_{k}-x^{*}\right\|^{2}-\left(\alpha_{k}^{0}-\beta\right)\left(\sum_{i=1}^{p} \alpha_{k}^{i}\left\|U_{i}\left(s_{k}\right)-s_{k}\right\|^{2}\right) .
$$

From the proof of Theorem 3.5, we have (3.25) holds, i.e.,

$$
\left\|y_{k+1}-y^{*}\right\|^{2} \leq\left\|t_{k}-y^{*}\right\|^{2}-\sigma_{k}\left(1-\mu-\sigma_{k}\right)\left\|t_{k}-T_{j(k)}\left(t_{k}\right)\right\|^{2} .
$$

Adding up the last two inequalities, and setting $l_{k}\left(x^{*}, y^{*}\right)=\left\|x_{k}-x^{*}\right\|^{2}+\left\|y_{k}-y^{*}\right\|^{2}$, we get

$$
\begin{aligned}
& l_{k+1}\left(x^{*}, y^{*}\right) \\
\leq & l_{k}\left(x^{*}, y^{*}\right)-\left(\alpha_{k}^{0}-\beta\right)\left(\sum_{i=1}^{p} \alpha_{k}^{i}\left\|U_{i}\left(s_{k}\right)-s_{k}\right\|^{2}\right)-\sigma_{k}\left(1-\mu-\sigma_{k}\right)\left\|t_{k}-T_{j(k)}\left(t_{k}\right)\right\|^{2} \\
& -\gamma_{k}\left[2\left\|A x_{k}-B y_{k}\right\|^{2}-\gamma_{k}\left(\left\|A^{*}\left(A x_{k}-B y_{k}\right)\right\|^{2}+\left\|B^{*}\left(A x_{k}-B y_{k}\right)\right\|^{2}\right)\right]
\end{aligned}
$$

by (3.8). We see that $\left\{l_{k}\left(x^{*}, y^{*}\right)\right\}$ is non-increasing and lower bounded by $0 .\left\{l_{k}\left(x^{*}, y^{*}\right)\right\}$ converges to some finite limit, say $l\left(x^{*}, y^{*}\right)$. Similar to the proof of Theorem 3.3, we have

$$
\lim _{k \rightarrow \infty}\left\|A x_{k}-B y_{k}\right\|=\lim _{k \rightarrow \infty}\left\|t_{k}-T_{j(k)}\left(t_{k}\right)\right\|=0
$$

and

$$
\lim _{k \rightarrow \infty}\left\|s_{k}-U_{i}\left(s_{k}\right)\right\|=0
$$

for each $1 \leq i \leq p$. Moreover, we have

$$
\lim _{k \rightarrow \infty}\left\|s_{k}-x_{k}\right\|=\lim _{k \rightarrow \infty}\left\|t_{k}-y_{k}\right\|=0 .
$$


It follows from (4.3), (4.4) and (4.5) that

$$
\lim _{k \rightarrow \infty}\left\|x_{k}-U_{i}\left(s_{k}\right)\right\|=\lim _{k \rightarrow \infty}\left\|y_{k}-T_{j(k)}\left(t_{k}\right)\right\|=0 .
$$

From

$$
\left\|x_{k+1}-x_{k}\right\| \leq \alpha_{k}^{0}\left\|s_{k}-x_{k}\right\|+\alpha_{k}^{1}\left\|U_{1}\left(s_{k}\right)-x_{k}\right\|+\cdots+\alpha_{k}^{p}\left\|U_{p}\left(s_{k}\right)-x_{k}\right\|
$$

and

$$
\left\|y_{k+1}-y_{k}\right\| \leq\left(1-\sigma_{k}\right)\left\|t_{k}-y_{k}\right\|+\sigma_{k}\left\|T_{j(k)}\left(t_{k}\right)-y_{k}\right\|,
$$

we have

$$
\lim _{k \rightarrow \infty}\left\|x_{k+1}-x_{k}\right\|=\lim _{k \rightarrow \infty}\left\|y_{k+1}-y_{k}\right\|=0,
$$

which infers that $\left\{x_{k}\right\}$ and $\left\{y_{k}\right\}$ are asymptotically regular and

$$
\lim _{k \rightarrow \infty}\left\|s_{k+1}-s_{k}\right\|=\lim _{k \rightarrow \infty}\left\|t_{k+1}-t_{k}\right\|=0 .
$$

Taking $(\tilde{x}, \tilde{y}) \in \omega_{\omega}\left(x_{k}, y_{k}\right)$, we have $(\tilde{x}, \tilde{y}) \in \omega_{\omega}\left(s_{k}, t_{k}\right)$. Combing the proof of Theorem 3.3 and Theorem 3.5, we have $\omega_{w}\left(x_{k}, y_{k}\right) \subseteq \Gamma$ and the sequence $\left\{\left(x_{k}, y_{k}\right)\right\}$ weakly converges to the solution of (1.8). This completes the proof.

Next, we propose another mixed self-adaptive parallel and cyclic iterative algorithm for solving MSECFP (1.8) of demicontractive operators.

Algorithm 4.3. Let $\left\{\alpha_{k}\right\} \subset[0,1]\left\{\sigma_{k}^{j}\right\}_{k=0}^{\infty} \subset[0,1](0 \leq j \leq r)$ be sequences such that $\Sigma_{j=0}^{r} \sigma_{k}^{j}=1$ for every $k \geq 0$. Take $x_{0} \in H_{1}, y_{0} \in H_{2}$, and calculate

$$
\left\{\begin{array}{l}
s_{k}=x_{k}-\gamma_{k} A^{*}\left(A x_{k}-B y_{k}\right), \\
x_{k+1}=\left(1-\alpha_{k}\right) s_{k}+\alpha_{k} U_{i(k)}\left(s_{k}\right), \\
t_{k}=y_{k}+\gamma_{k} B^{*}\left(A x_{k}-B y_{k}\right), \\
y_{k+1}=\sigma_{k}^{0} t_{k}+\sigma_{k}^{1} T_{1}\left(t_{k}\right)+\cdots+\sigma_{k}^{r} T_{r}\left(t_{k}\right),
\end{array}\right.
$$

where $i(k)=k(\bmod p)+1$ and the stepsize $\gamma_{k}$ is chosen as (3.2).

Using a similar argument in the proof of Theorem 4.2, we conclude that the following result.

Theorem 4.4. Let $\left\{U_{i}, \quad 1 \leq i \leq p\right\}: H_{1} \rightarrow H_{1}$ and $\left\{T_{j}, \quad 1 \leq j \leq r\right\}: H_{2} \rightarrow H_{2}$ be $\beta_{i}$-demicontractive and $\mu_{j}$-demicontractive operators, respectively. Assume that $U_{i}-I(1 \leq i \leq p), T_{j}-I(1 \leq j \leq r)$ are demiclosed at origin and $\Gamma$ is nonempty. Let $\beta=\max _{1 \leq i \leq p}\left\{\beta_{i}\right\}$ and $\mu=\max _{1 \leq j \leq r}\left\{\mu_{j}\right\}$. Then the sequence $\left\{\left(x_{k}, y_{k}\right)\right\}$ generated by Algorithm 4.3 weakly converges to a solution $\left(x^{*}, y^{*}\right)$ of (1.8), provided that $0<\liminf _{k \rightarrow \infty} \alpha_{k} \leq \limsup _{k \rightarrow \infty} \alpha_{k}<1-\beta, \liminf _{k \rightarrow \infty} \sigma_{k}^{0}>\mu$ and $\liminf _{k \rightarrow \infty} \sigma_{k}^{i}>0$ for all $1 \leq j \leq$ r. Moreover, $\left\|A x_{k}-B y_{k}\right\| \rightarrow 0,\left\|x_{k}-x_{k+1}\right\| \rightarrow 0$ and $\left\|y_{k}-y_{k+1}\right\| \rightarrow 0$ as $k \rightarrow \infty$.

Remark 4.5. For the particular case " $\mathrm{p}=\mathrm{r}=1$ ", Algorithm 4.1 and Algorithm 4.3 become mixed iterative algorithms to solve SECFP (1.5) governed by demicontractive operators without prior knowledge of operator norms.

From Lemma 2.8, we have the following results on MSECFP (1.8) of strictly pseudocontractive operators. 
Corollary 4.6. Let $\left\{U_{i}, \quad 1 \leq i \leq p\right\}: H_{1} \rightarrow H_{1}$ and $\left\{T_{j}, \quad 1 \leq j \leq r\right\}: H_{2} \rightarrow H_{2}$ be $\beta_{i}$-strictly pseucontractive and $\mu_{j}$-strictly pseucontractive operators, respectively. Assume that $\Gamma$ is nonempty. Let $\beta=\max _{1 \leq i \leq p}\left\{\beta_{i}\right\}$ and $\mu=\max _{1 \leq j \leq r}\left\{\mu_{j}\right\}$. Then the sequence $\left\{\left(x_{k}, y_{k}\right)\right\}$ generated by Algorithm 4.1 weakly converges to a solution $\left(x^{*}, y^{*}\right)$ of (1.8), provided that $\liminf _{k \rightarrow \infty} \alpha_{k}^{0}>\beta, \liminf _{k \rightarrow \infty} \alpha_{k}^{i}>0$ for all $1 \leq i \leq p$ and $0<\liminf _{k \rightarrow \infty} \sigma_{k} \leq \limsup _{k \rightarrow \infty} \sigma_{k}<1-\mu$. Moreover, $\left\|A x_{k}-B y_{k}\right\| \rightarrow 0,\left\|x_{k}-x_{k+1}\right\| \rightarrow 0$ and $\left\|y_{k}-y_{k+1}\right\| \rightarrow 0$ as $k \rightarrow \infty$.

Corollary 4.7. Let $\left\{U_{i}, \quad 1 \leq i \leq p\right\}: H_{1} \rightarrow H_{1}$ and $\left\{T_{j}, \quad 1 \leq j \leq r\right\}: H_{2} \rightarrow H_{2}$ be $\beta_{i}$-strictly pseucontractive and $\mu_{j}$-strictly pseucontractive operators, respectively. Assume that $\Gamma$ is nonempty. Let $\beta=\max _{1 \leq i \leq p}\left\{\beta_{i}\right\}$ and $\mu=\max _{1 \leq j \leq r}\left\{\mu_{j}\right\}$. Then the sequence $\left\{\left(x_{k}, y_{k}\right)\right\}$ generated by Algorithm 4.3 weakly converges to a solution $\left(x^{*}, y^{*}\right)$ of (1.8), provided that $0<\liminf _{k \rightarrow \infty} \alpha_{k} \leq \limsup _{k \rightarrow \infty} \alpha_{k}<1-\beta$, $\liminf _{k \rightarrow \infty} \sigma_{k}^{0}>\mu$ and $\liminf _{k \rightarrow \infty} \sigma_{k}^{i}>0$ for all $1 \leq j \leq r$. Moreover, $\left\|A x_{k}-B y_{k}\right\| \rightarrow 0,\left\|x_{k}-x_{k+1}\right\| \rightarrow 0$ and $\left\|y_{k}-y_{k+1}\right\| \rightarrow 0$ as $k \rightarrow \infty$.

\section{NUMERICAL EXPERIMENTS}

In this section, we provide some numerical experiments and show the performance of the proposed self-adaptive iterative algorithms with stepsize (3.2) for solving the MSECFP (1.8). All the codes are written in MATLAB and are performed on a personal Lenovo computer with Pentium(R) Dual-Core CPU @ 2.4GHz and RAM 2.00GB.

In this part, we take the following experiment parameters $\rho_{k}=1.95$ in all iterative algorithms, and the stopping criteria is $f(x, y)<10^{-5}$. Here function $f(x, y)$ measures the distance given as follows

$$
f(x, y)=\Sigma_{i=1}^{p}\left\|x-U_{i} x\right\|+\Sigma_{j=1}^{r}\left\|y-T_{j} y\right\| .
$$

Let $R^{n}$ be $n$ dimensional Euclidean space with inner product

$$
\langle x, y\rangle=x_{1} y_{1}+\cdots+x_{n} y_{n}
$$

and norm $\|x\|=\sqrt{x_{1}^{2}+\cdots+x_{n}^{2}}$ for all $x=\left(x_{1}, \cdots, x_{n}\right)^{T}, y=\left(y_{1}, \cdots, y_{n}\right)^{T} \in R^{n}$.

Example 5.1. Define operators $U_{i}: R^{2} \rightarrow R^{2}$ and $T_{i}: R^{2} \rightarrow R^{2}(i=1,2)$ as follows:

$$
U_{1}(x)=\left(z_{1}, z_{2}\right)^{T}, U_{2}(x)=\left(x_{1}, x_{2}\right)^{T}, T_{1}(x)=\left(\frac{x_{1}}{3}, \frac{x_{2}}{3}\right)^{T}, T_{2}(x)=\left(\frac{x_{1}}{2}, \frac{x_{2}}{2}\right)^{T},
$$

where $x=\left(x_{1}, x_{2}\right)^{T}$ and

$$
z_{i}=\left\{\begin{array}{ll}
x_{i}, & x_{i}<0, \\
-2 x_{i}, & x_{i} \geq 0,
\end{array} \quad(i=1,2) .\right.
$$

Note that $U_{1}$ is $\frac{1}{3}$-demicontractive and $U_{2}, T_{1}, T_{2}$ are nonexpansive operators. Obviously,

$$
F\left(U_{1}\right) \cap F\left(U_{2}\right)=\left\{\left(x_{1}, x_{2}\right)^{T}: x_{1} \leq 0, x_{2} \leq 0\right\}, \quad F\left(T_{1}\right) \cap F\left(T_{2}\right)=\left\{(0,0)^{T}\right\} .
$$

Let

$$
A=\left(\begin{array}{ll}
2 & 6 \\
2 & 4
\end{array}\right), B=\left(\begin{array}{ll}
3 & 8 \\
1 & 1
\end{array}\right) .
$$

We consider the following MSECFP:

finding $x^{*} \in F\left(U_{1}\right) \cap F\left(U_{2}\right), y^{*} \in F\left(T_{1}\right) \cap F\left(T_{2}\right)$, such that $A x^{*}=B y^{*}$. 
TABLE 1. Numerical results for solving Example 5.1 with different iterative algorithms.

\begin{tabular}{cccc}
\multicolumn{4}{c}{$x_{0}=(-1,5)^{T}, y_{0}=(3,1)^{T}$} \\
\hline & $\mathrm{k}$ & $x_{k}$ & $y_{k}$ \\
\hline Algorithm 3.1 & 108 & $(-0.7707,-0.1919)^{T} \times 10^{-5}$ & $(-0.1973,0.8577)^{T} \times 10^{-6}$ \\
Algorithm 3.4 & 81 & $(0.1390,-0.3408)^{T} \times 10^{-5}$ & $(-0.1571,-0.3707)^{T} \times 10^{-5}$ \\
Algorithm 4.1 & 125 & $(-0.5490,0)^{T} \times 10^{-5}$ & $(-0.1556,-0.3228)^{T} \times 10^{-5}$ \\
Algorithm 4.3 & 69 & $(0.0262,-0.4602)^{T} \times 10^{-5}$ & $(-0.1983,-0.4614)^{T} \times 10^{-5}$ \\
\hline
\end{tabular}

We apply the proposed self-adaptive simultaneous iterative Algorithm 3.1, cyclic iterative Algorithm 3.4, and two mixed iterative Algorithm 4.1 and 4.3 to solve Example 5.1. We take initial points $x_{0}=$ $(-1,5)^{T}, y_{0}=(3,1)^{T}$. In self-adaptive simultaneous iterative Algorithm 3.1, we take $\alpha_{k}^{0}=\alpha_{k}^{1}=\alpha_{k}^{2}=$ $\sigma_{k}^{0}=\sigma_{k}^{1}=\sigma_{k}^{2}=\frac{1}{3}$. In self-adaptive cyclic iterative Algorithm 3.4, we take $\alpha_{k}=\sigma_{k}=\frac{1}{3}$. In self-adaptive mixed iterative Algorithm 4.1 and Algorithm 4.3, we take $\alpha_{k}^{0}=\alpha_{k}^{1}=\alpha_{k}^{2}=\sigma_{k}=\frac{1}{3}$ and $\alpha_{k}=\sigma_{k}^{0}=\sigma_{k}^{1}=$ $\sigma_{k}^{2}=\frac{1}{3}$, respectively. The numerical results are given in Table 1 . Denote $x_{k}$ and $y_{k}$ as the kth iterative sequences.

Example 5.2. Define the operators $U_{i}: R^{3} \rightarrow R^{3}$ and $T_{i}: R^{2} \rightarrow R^{2}(i=1,2)$ as follows:

$$
U_{1}(x)=\left(z_{1}, z_{2}, z_{3}\right)^{T}, U_{2}(x)=\left(x_{1}, x_{2}, x_{3}\right)^{T}, T_{1}(y)=\left(w_{1}, w_{2}\right)^{T}, T_{2}(y)=\left(\frac{y_{1}}{2}, \frac{y_{2}}{2}\right)^{T},
$$

where $x=\left(x_{1}, x_{2}, x_{3}\right)^{T}, y=\left(y_{1}, y_{2}\right)^{T}$ and

$$
z_{i}=\left\{\begin{array}{ll}
x_{i}, & x_{i}<0, \\
-2 x_{i}, & x_{i} \geq 0,
\end{array} \quad(i=1,2,3), \quad w_{j}=\left\{\begin{array}{ll}
y_{j}, & y_{j}<0, \\
-3 y_{j}, & y_{j} \geq 0,
\end{array} \quad(j=1,2) .\right.\right.
$$

Note that $U_{1}$ is $\frac{1}{3}$-demicontractive, $T_{1}$ is $\frac{1}{2}$-demicontractive and $U_{2}, T_{2}$ are nonexpansive operators. Obviously,

$$
F\left(U_{1}\right) \cap F\left(U_{2}\right)=\left\{\left(x_{1}, x_{2}\right)^{T}: x_{1} \leq 0, x_{2} \leq 0\right\}, F\left(T_{1}\right) \cap F\left(T_{2}\right)=\left\{(0,0)^{T}\right\}
$$

Let

$$
A=\left(\begin{array}{ccc}
1 & -6 & -1 \\
2 & 2 & 4
\end{array}\right), B=\left(\begin{array}{cc}
3 & -8 \\
-1 & 1
\end{array}\right)
$$

We consider the following MSECFP:

$$
\text { finding } x^{*} \in F\left(U_{1}\right) \cap F\left(U_{2}\right), y^{*} \in F\left(T_{1}\right) \cap F\left(T_{2}\right) \text {, such that } A x^{*}=B y^{*} .
$$

We apply the proposed self-adaptive simultaneous iterative Algorithm 3.1, cyclic iterative Algorithm 3.4, and two mixed iterative Algorithm 4.1 and 4.3 to solve Example 5.2. We take different initial points $x_{0}=(5,-6,-1)^{T}, y_{0}=(3,0)^{T}$ and $x_{0}=(3,4,-1)^{T}, y_{0}=(-3,0)^{T}$. In self-adaptive simultaneous iterative Algorithm 3.1, we take $\alpha_{k}^{0}=\alpha_{k}^{1}=\alpha_{k}^{2}=\frac{1}{3}, \sigma_{k}^{0}=\frac{1}{2}$ and $\sigma_{k}^{1}=\sigma_{k}^{2}=\frac{1}{4}$. In self-adaptive cyclic iterative Algorithm 3.4, we take $\alpha_{k}=\frac{1}{3}$ and $\sigma_{k}=\frac{1}{2}$. In self-adaptive mixed iterative Algorithm 4.1 and Algorithm 4.3, we take $\alpha_{k}^{0}=\alpha_{k}^{1}=\alpha_{k}^{2}=\frac{1}{3}, \sigma_{k}=\frac{1}{2}$ and $\alpha_{k}=\frac{1}{3}, \sigma_{k}^{0}=\frac{1}{2}, \sigma_{k}^{1}=\sigma_{k}^{2}=\frac{1}{4}$, respectively. The numerical results are given in Table 2-3. Denote $x_{k}$ and $y_{k}$ as the kth iterative sequences. 
TABLE 2. Numerical results for solving Example 5.2 with different iterative algorithms.

\begin{tabular}{cccc}
\multicolumn{4}{c}{$x_{0}=(5,-6,-1)^{T}, y_{0}=(3,0)^{T}$} \\
\hline & $\mathrm{k}$ & $x_{k}$ & $y_{k}$ \\
\hline Algorithm 3.1 & 173 & $(0,-0.2425,-0.0097)^{T} \times 10^{-4}$ & $(-0.4193,-0.8367)^{T} \times 10^{-5}$ \\
Algorithm 3.4 & 327 & $(0.0017,-0.2485,-0.0493)^{T} \times 10^{-4}$ & $(-0.8625,-0.1308)^{T} \times 10^{-5}$ \\
Algorithm 4.1 & 307 & $(0,-0.2960,-0.0381)^{T} \times 10^{-4}$ & $(-0.8566,-0.4243)^{T} \times 10^{-5}$ \\
Algorithm 4.3 & 147 & $(-0.0014,-0.2162,-0.0375)^{T} \times 10^{-4}$ & $(-0.6753,-0.2237)^{T} \times 10^{-5}$ \\
\hline
\end{tabular}

TABLE 3. Numerical results for solving Example 5.2 with different iterative algorithms.

\begin{tabular}{cccc}
\multicolumn{4}{c}{$x_{0}=(3,4,-1)^{T}, y_{0}=(-3,0)^{T}$} \\
\hline & $\mathrm{k}$ & $x_{k}$ & $y_{k}$ \\
\hline Algorithm 3.1 & 144 & $(0,-0.2402,-0.0096)^{T} \times 10^{-4}$ & $(-0.4153,-0.8287)^{T} \times 10^{-5}$ \\
Algorithm 3.4 & 111 & $(-0.2759,-0.2050,0.1505)^{T} \times 10^{-5}$ & $(0.1863,-0.4865)^{T} \times 10^{-5}$ \\
Algorithm 4.1 & 87 & $(0,0,0)^{T}$ & $(-0.5918,-0.5465)^{T} \times 10^{-5}$ \\
Algorithm 4.3 & 191 & $(-0.0012,-0.3678,0.1122)^{T} \times 10^{-5}$ & $(-0.0061,-0.6484)^{T} \times 10^{-5}$ \\
\hline
\end{tabular}

\section{Acknowledgements}

This paper was supported by the Scientific Research Project of Tianjin Municipal Education Commission under Grant No. 2018KJ253 and Science and Technology Planning Project of Shandong Higher Education Institution under Grant No. J17KB122.

\section{REFERENCES}

[1] Y. Censor, T. Elfving, N. Kopf, T. Bortfeld, The multiple-sets split feasibility problem and its applications for inverse problems, Inverse Probl. 21 (2005), 2071-2084.

[2] Y. Censor, T. Elfving, A multiprojection algorithm using Bregman projections in a product space, Numer. Algorithms 8 (1994), 221-239.

[3] C. Byrne, Iterative oblique projection onto convex subsets and the split feasibility problem, Inverse Probl. 18 (2002), 441-453.

[4] Q. Yang, The relaxed CQ algorithm solving the split feasibility problem, Inverse Probl. 20 (2004), 1261-1266.

[5] B. Qu, N. Xiu, A note on the CQ algorithm for the split feasibility problem, Inverse Probl. 21 (2005), 1655-1665.

[6] H.H. Bauschke, J.M. Borwein, On projection algorithms for solving convex feasibility problems, SIAM Rev. 38 (1996), 367-426.

[7] H. Chen, Y. Wang, G. Wang, Strong convergence of extragradient method for generalized variational inequalities in Hilbert space, J. Inequal. Appl. 2014 (2014), 223.

[8] E. Masad, S. Reich, A note on the multiple-set split convex feasibility problem in Hilbert space, J. Nonlinear Convex Anal. 8 (2007), 367-371.

[9] X. Qin, J.C. Yao, Projection splitting algorithms for nonself operators, J. Nonlinear Convex Anal. 18 (2017), 925-935.

[10] H.K. Xu, A variable Krasnosel'skii-Mann algorithm and the multiple-set split feasibility problem, Inverse Probl. 22 (2006), 2021-2034.

[11] X. Qin, S.Y. Cho, L. Wang, Strong convergence of an iterative algorithm involving nonlinear mappings of nonexpansive and accretive type, Optimization, 67 (2018), 1377-1388.

[12] H. Zhang, Y. Wang, A new CQ method for solving split feasibility problem, Front. Math. China 5 (2010), 37-46.

[13] Y. Censor, A. Segal, The split common fixed point problem for directed operators, J. Convex Anal. 16 (2009), 587-600. 
[14] M. Eslamian, G.Z. Eskandani, M. Raeisi, Split common null point and common fixed point problems between Banach spaces and Hilbert spaces, Mediterr. J. Math. 14 (2017), Article ID 119.

[15] B. Liu, B. Qu, N. Zheng, A successive projection algorithm for solving the multiple-sets split feasibility problem, Numer. Funct. Anal. Optim. 35 (2014), 1459-1466.

[16] X. Qin, A. Petrusel, J.C. Yao, CQ iterative algorithms for fixed points of nonexpansive mappings and split feasibility problems in Hilbert spaces, J. Nonlinear Convex Anal. 19 (2018), 157-165.

[17] S. He, H. Tian, H.K. Xu, The selective projection method for convex feasibility and split feasibility problems, J. Nonlinear Convex Anal. 19 (2018), 1199-1215.

[18] Q.L. Dong, H.B. Yuan, Y.J. Cho, Th.M. Rassias, Modified inertial Mann algorithm and Inertial CQ-algorithm for nonexpansive mappings, Optim. Lett. 12 (2018), 87-102.

[19] Q.L. Dong, Y.C. Tang, Y.J. Cho, Th.M. Rassias, "Optimal" choice of the step length of the projection and contraction methods for solving the split feasibility problem, J. Glob. Optim. 71 (2018), 341-360.

[20] L. Yang, F. Zhao, J.K. Kim, The split common fixed point problem for demicontractive mappings in Banach spaces, J. Comput. Anal. Appl. 22 (2017), 858-863.

[21] Y. Yao, Y.C. Liou, J.C. Yao, Split common fixed point problem for two quasi-pseudocontractive operators and its algorithm construction, Fixed Point Theory Appl. 2015 (2015), Article ID 127.

[22] S.Y. Cho, X. Qin, J.C. Yao, Y. Yao, Viscosity approximation splitting methods for monotone and nonexpansive operators in Hilbert spaces, J. Nonlinear Convex Anal. 19 (2018), 251-264.

[23] F. Wang, H.K. Xu, Cyclic algorithms for split feasibility problems in Hilbert spaces. Nonlinear Anal. 74 (2011), $4105-$ 4111.

[24] Y.C. Tang, L.W. Liu, Several iterative algorithms for solving the split common fixed point problem of directed operators with applications, Optimization, 65 (2016), 53-65.

[25] A. Moudafi, The split common fixed point problem for demicontractive mappings, Inverse Probl. 26 (2010), Article ID 055007.

[26] Y.C. Tang, J.G. Peng, L.W. Liu, A cyclic algorithm for the split common fixed point problem of demicontractive mappings in Hilbert spaces, Math. Modelling Anal. 17 (2012), 457-466.

[27] A. Moudafi, Alternating CQ-algorithms for convex feasibility and split fixed-point problems, J. Nonlinear Convex Anal. 15 (2014), 809-818.

[28] A. Moudafi, E. Al-Shemas, Simultaneous iterative methods for split equality problems and application, Trans. Math. Program. Appl. 1 (2013), 1-11.

[29] H. Attouch, J. Bolte, P. Redont, A. Soubeyran, Alternating proximal algorithms for weakly coupled minimization problems, Applications to dynamical games and PDEs, J. Convex Anal. 15 (2008), 485-506.

[30] Y. Censor, T. Bortfeld, B. Martin, A. Trofimov, A unified approach for inversion problems in intensity-modulated radiation therapy, Phys. Med. Biol. 51 (2006), 2353-2365.

[31] D.M. Giang, J.J. Strodiot, V.H. Nguyen, Strong convergence of an iterative method for solving the multiple-set split equality fixed point problem in a real Hilbert space, Rev. R. Acad. Cienc. Exactas Fis. Nat. Ser. A Math. RACSAM 111 (2017), 983-998.

[32] J. Wang, Y. Wang, Strong convergence of a cyclic iterative algorithm for split common fixed-point problems of demicontractive mappings, J. Nonlinear Var. Anal. 2 (2018), 295-303.

[33] Y. Wu, R. Chen, L.Y. Shi, Split equality problem and multiple-sets split equality problem for quasi-nonexpansive multivalued mappings, J. Inequal. Appl. 2014 (2014), Article ID 428.

[34] J. Zhao, Solving split equality fixed-point problem of quasi-nonexpansive mappings without prior knowledge of operators norms, Optimization 64 (2015), 2619-2630.

[35] J. Zhao, S. He, Solving the general split common fixed-point problem of quasi-nonexpansive mappings without prior knowledge of operator norms, Filomat 31 (2017), 559-573.

[36] H. Zegeye, N. Shahzad, Convergence of Mann's type iteration method for generalized asymptotically nonexpansive mappings, Comput. Math. Appl. 62 (2011), 4007-4014.

[37] F.E. Browder, W.V. Petryshyn, Construction of fixed points of nonlinear mappings in Hilbert spaces, J. Math. Anal. Appl. 20(1967), 197-228. 